\title{
Optimal Power Allocation in Multi-Hop Cooperative Network Using Non-Regenerative Relaying Protocol
}

\author{
Muhammad H.D. Khan, Mohammed S. Elmusrati and Reino Virrankoski \\ Communication and System Engineering Group \\ University of Vaasa, Vaasa, Finland \\ hazandanish@yahoo.com,moel@uwasa.fi,rvir@uwasa.fi
}

\begin{abstract}
Cooperative transmission is one of the promising techniques in wireless communication systems that enables the cooperating node in a wireless sensor network to share their radio resources by employing a distributed transmission and processing operation. This technique offers significant diversity gains as several cooperating nodes forward source node's data to the destination node over independently fading channels. The benefits offered by cooperative transmission can only be exploited fully if the power is allocated between source and cooperating nodes in an optimal manner instead of equal power allocation (EPA). Therefore, in this paper using moment generating function (MGF) approach a closed-form expression of probability of error has been derived for multi-hop cooperative network employing amplify-and-forward (AF) over rayleigh fading channel. Moreover, using two different network scenarios, optimal power allocation (OPA) scheme has been further investigated on the basis of channel link qualities between the communicating nodes. Numerical and simulation results validate the performance improvement of OPA over EPA and further the improvement due to relay location in the cooperative network.
\end{abstract}

Keywords-Cooperative transmission, amplify-and-forward, maximal ratio combining, optimal power allocation. moment generation function

\section{INTRODUCTION}

In recent years, many communication systems have been designed to enhance the performance in terms of link reliability, throughput rates, and network capacity. But one of the major technical challenges in wireless communication is of multipath fading which arises because of the scattering and reflection of the transmitted signal. This phenomenon induces channel impairments but when properly exploited, it can also be used to provide spatial diversity gains.

Cooperative transmission is one such technique, in which spatial diversity is achieved by sharing the single antennas of cooperating nodes to form a virtual antenna array, as proposed in [1][2]. The cooperating nodes along with the source node transmit same information signal over independent paths and at the receiver these multiple versions of the same signal help to achieve a higher diversity gain. In cooperative relay networks, one of the most popular relaying protocols is amplify-andforward (AF) protocol which simply amplifies the received signals from the source node and then forwards it to the destination node. The practical advantage of the AF relaying protocol lies in its simple execution and low cost implementation. In a cooperative relay network, the power available at the communicating nodes is limited. Therefore, it is very crucial to utilize this power in an efficient manner.
In order to implement and effectuate the relaying schemes in the cooperative network, one of the main tasks is to develop an optimal power allocation (OPA) strategy. The OPA strategy is an effective way to save the transmission power and enhance the performance of relay networks. The main objective is to find the optimal power allocation of the source node and relays involved to maximize the QoS performance at the destination node with a total power constraint.

The optimal power allocation of a two-hop AF cooperation network was examined in [3] and [4] for Rayleigh fading, but they only considered a single relay network. In [5], the power allocation has been discussed for a multi-node network but decode-and-forward (DF) relaying protocol has been employed over Rician fading channel. Moreover in [6] [7] and [8], OPA has been derived for AF dual-hop network with arbitrary number of relays over Rayleigh fading. In this paper OPA has been derived for AF multi-hop network with multiple relays using moment generation function (MGF) method. The closedform expression for the probability of error is derived using the Lagrangian multiplier method.

\section{MULTI-HOP COOPERATIVE NETWORK}

\section{A. System Model (2-Hop Scenario)}

For this scenario the network configuration is shown below, Fig 1. The network comprises of three nodes; a source node $S$, destination node $D$ and a relay node $R$. Let $G_{S D}, G_{S R}$, and $G_{R D}$ represent the source-destination, source-relay and relaydestination channel coefficients, respectively. The system model is designed based on assumptions that a Maximum Ratio Combining (MRC) is used by the receiver as a detector, and Amplify and Forward (AF) is used as a relaying strategy.

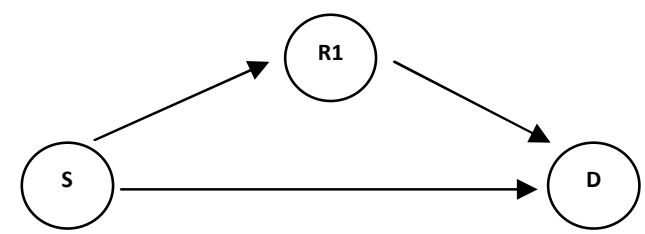

Figure 1. Power allocation with AF involving 1-Relay

In the first phase the source node $S$ broadcasts the data $X$ with its transmission power $P_{S}$ to both relay and destination node, as shown in the next figure. The channel gains for the sourcerelay and from source- destination links are $G_{S R}$ and $G_{S D}$ respectively. The AWGN present at the relay and destination nodes will be $n_{S R}$ and $n_{S D}$ respectively. The equation of received signals in the phase-I at the relay and destination will be expressed as $Y_{R}$ and $Y_{D}$ respectively. 


$$
\begin{aligned}
& \mathrm{Y}_{\mathrm{D}}[\mathrm{n}]=\sqrt{P_{S}} \quad G_{S D} \cdot \mathrm{X}[\mathrm{n}]+\mathrm{n}_{\mathrm{SD}}[\mathrm{n}] \\
& \mathrm{Y}_{\mathrm{R}}[\mathrm{n}]=\sqrt{P_{S}} G_{S R} \cdot \mathrm{X}[\mathrm{n}]+\mathrm{n}_{\mathrm{SR}}[\mathrm{n}]
\end{aligned}
$$

In phase-II, the relay amplifies the received signal $Y_{R}$ and then forwards it to the destination with its amplifier gain $\beta_{R}$. Here, $P_{R}$ is the transmission power of the relay node. $G_{R D}$ is the channel gain for relay-destination link and $n_{R D}$ is the noise at the receiver in phase-II. Now the signal received at the destination from the relay is:

$$
\mathrm{Y}_{\mathrm{R}}^{\prime}[\mathrm{n}]=\beta_{\mathrm{R}} \cdot \mathrm{Y}_{\mathrm{R}}[\mathrm{n}] \cdot G_{R D}+\mathrm{n}_{\mathrm{RD}}[\mathrm{n}]
$$

Relay gain $\beta_{R}$ is used to properly fine-tune the power at the relay to reduce variations in the relay-destination link. The relaying node's amplifier can provide a maximum gain defined by the following expressions:

$$
\beta_{\mathrm{R}}=\sqrt{\frac{P_{R}}{P_{S}\left|G_{S R}\right|^{2}+N_{0}}}
$$

Since, amplify-and-forward protocol may induce some noise amplification but the MRC detector employed is quite competitive therefore with the help of its weights it can compensate effect of induced noise. The output of the MRC detector at the destination node will be:

$$
\begin{gathered}
\mathrm{Y}_{\mathrm{MRC}}[\mathrm{n}]=\alpha_{0}\left(\sqrt{P_{S}} \cdot G_{S D} \cdot \mathrm{X}[\mathrm{n}]+\mathrm{n}_{\mathrm{SD}}[\mathrm{n}]\right)+ \\
\alpha_{1}\left(\beta_{\mathrm{R}} \cdot \mathrm{Y}_{\mathrm{R}}[\mathrm{n}] \cdot G_{R D}+\mathrm{n}_{\mathrm{RD}}[\mathrm{n}]\right)
\end{gathered}
$$

In the above equation, $\alpha_{0}$ and $\alpha_{1}$ are the weights of the maximal-ratio-combiner. These combining weight compensate for the effects of likely incorrect decisions and are therefore chosen accordingly.

$$
\begin{gathered}
\alpha_{0}=\frac{\sqrt{P_{S}} G_{S D}{ }^{*}}{N_{0}} \\
\alpha_{1}=\frac{\sqrt{P_{S}} \cdot \beta_{R} \cdot G_{S R}{ }^{*} \cdot G_{R D}{ }^{*}}{N_{0}}
\end{gathered}
$$

\section{B. System Model (3-Hop Scenario)}

For this scenario the network configuration is shown below, Fig 2. The network comprises of four nodes; a source node $S$, destination node $D$, a relay node $R 1$ and a relay node $R 2$. Let $G_{S D}, G_{S R 1}, G_{R I R 2}$ and $G_{R 2 D}$ represent the source-destination, source-relay1, relay1-relay2 and relay2-destination channel coefficients, respectively. The system model is designed based on assumptions that a Maximum Ratio Combining (MRC) is used by the receiver as a detector, and Amplify and Forward $(\mathrm{AF})$ is used as a relaying strategy.

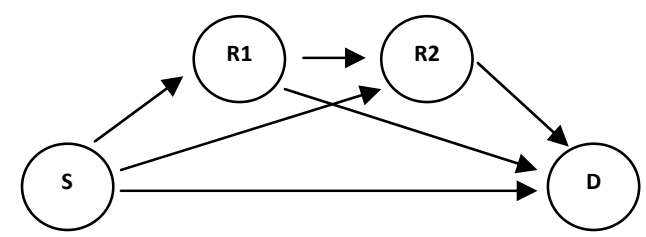

Figure 2. Power allocation with AF involving 2-Relays
In the first phase the source node $S$ broadcasts the data $X$ with its transmission power $P_{S}$ to both relay and destination node, as shown in the next figure. The channel gains for the source-relay1 and from source- relay2 links are $G_{S R I}$ and $G_{S R 2}$ respectively. The AWGN present at the relay 1 and relay 2 nodes will be $n_{S R 1}$ and $n_{S R 2}$ respectively. The equation of received signals in the phase-I at the relay 1 and relay 2 will be expressed as $Y_{R 1}$ and $Y^{\prime}{ }_{R 2}$ respectively

$$
\begin{aligned}
& \mathrm{Y}_{\mathrm{R} 1}[\mathrm{n}]=\sqrt{P_{S}} \quad G_{S R 1} \cdot \mathrm{X}[\mathrm{n}]+\mathrm{n}_{\mathrm{SR} 1}[\mathrm{n}] \\
& \mathrm{Y}_{\mathrm{R} 2}^{\prime}[\mathrm{n}]=\sqrt{P_{S}} G_{S R 2} \cdot \mathrm{X}[\mathrm{n}]+\mathrm{n}_{\mathrm{SR} 2}[\mathrm{n}]
\end{aligned}
$$

After Phase-II, the relay2 node has received two signals $Y_{R 2}^{\prime}$ and $Y_{R 2}$, first one was in phase-I (broadcasting) and the second one was forwarded by relayl with its amplification factor $\beta_{R I}$ in phase-II. $G_{R I R 2}$ is the channel gain for relay1-relay2 link and $n_{R I R 2}$ is the noise at the relay node2 in phase-II. But since the link between source-relay2 is weak as compared to the link between relay1 and relay 2 so the node relay 2 discards the signal $Y_{R 2}^{\prime}$ and the received signal $Y_{R 2}$ is defined as:

$$
\mathrm{Y}_{\mathrm{R} 2}[\mathrm{n}]=\beta_{\mathrm{R} 1} \cdot \mathrm{Y}_{\mathrm{R} 1}[\mathrm{n}] \cdot G_{R 1 R 2}+\mathrm{n}_{\mathrm{R} 1 \mathrm{R} 2}[\mathrm{n}]
$$

Relay gains $\beta_{R 1}$ and $\beta_{R 2}$ are used to properly fine-tune the powers at the corresponding relays to reduce variations in the source-relay and relay-destination links. The relaying node's amplifier can provide a maximum gain defined by the following expressions:

$$
\begin{gathered}
\beta_{\mathrm{R} 1}=\sqrt{\frac{P_{R 1}}{P_{S}\left|G_{S R 1}\right|^{2}+N_{0}}} \\
\beta_{\mathrm{R} 2}=\sqrt{\frac{P_{R 2}}{P_{R 1}\left|G_{R 1 R 2}\right|^{2}+N_{0}}}
\end{gathered}
$$

Since, amplify-and-forward protocol may induce some noise amplification but the MRC detector employed is quite competitive therefore with the help of its weights it can compensate effect of induced noise. The output of the MRC detector at the destination node will be:

$$
\begin{aligned}
& \mathrm{Y}_{\mathrm{MRC}}[\mathrm{n}]=\alpha_{0}\left(\sqrt{P_{S}} \cdot G_{S D} \cdot \mathrm{X}[\mathrm{n}]+\mathrm{n}_{\mathrm{SD}}[\mathrm{n}]\right)+ \\
& \alpha_{1}\left(\beta_{\mathrm{R} 2} \cdot \mathrm{Y}_{\mathrm{R} 2}[\mathrm{n}] . G_{R 2 D}+\mathrm{n}_{\mathrm{R} 2 \mathrm{D}}[\mathrm{n}]\right)
\end{aligned}
$$

In the above equation, $\alpha_{0}$ and $\alpha_{1}$ are the weights of the maximal-ratio-combiner. These combining weight compensate for the effects of likely incorrect decisions and are therefore chosen accordingly. Here $\alpha_{1}$, is the weight of the signals which is being forwarded by the relays placed in a linear topology.

$$
\begin{gathered}
\alpha_{0}=\frac{\sqrt{P_{S}} G_{S D}{ }^{*}}{N_{0}} \\
\alpha_{1}=\frac{\sqrt{P_{S}} \cdot \beta_{R 1} \cdot \beta_{R 2} \cdot G_{S R 1}{ }^{*} \cdot G_{R 1 R 2}{ }^{*} \cdot G_{R 2 D}{ }^{*}}{N_{0}}
\end{gathered}
$$




\section{OPTIMAL POWER ALLOCATION}

\section{A. 2-Hop Scenario}

The probability of bit error will be calculated using Moment Generating Function (MGF) approach. Since, it is a very helpful and simple tool for performance analysis of any modulation scheme in a fading scenario. In our case the modulation scheme is M-PSK.

$$
\begin{gathered}
P_{e}=\frac{1}{\pi} \int_{0}^{\left(\frac{N-1}{N}\right) \pi} \prod_{n=0}^{1} N_{\gamma_{n}}\left(\frac{g_{P S K}}{\sin ^{2}}\right) d \theta \\
N_{\gamma_{n}}\left(\frac{1}{\sin ^{2}}\right)=\left(1+\frac{g_{P S K}}{\sin ^{2}} \gamma_{n}\right)^{-1} \\
P_{e}=N_{o}^{2}\left[\frac{P_{e}=\left(\gamma_{0} \cdot \gamma_{1}\right)^{-1}}{P_{S} \cdot \sigma_{S D}^{2} \cdot \sigma_{S R}^{2}}+\frac{1}{P_{S} \cdot P_{R} \cdot \sigma_{S D}^{2} \cdot \sigma_{R D}^{2}}\right]
\end{gathered}
$$

Since the outage probability has been formulated for this configuration, an effort is made to minimize this outage probability. This will be done keeping in mind the constraint of total transmission power. This optimization problem can be expressed as:

$$
\begin{array}{cl}
\text { Minimize } & P_{e}=\frac{1}{\pi} \int_{0}^{\frac{\pi}{2}} \prod_{n=0}^{1} N_{\gamma_{n}}\left(\frac{1}{\sin ^{2}}\right) d \theta \\
& \text { Subject to } P_{S}+P_{R} \bullet P_{T}
\end{array}
$$

Now as we have formulated the constrained optimization problem of this network configuration, we proceed with its solution. One of the most important methods to get a closed form expression of a constrained optimization problem is the Lagrange Method. The Lagrange cost function of this problem is defined as:

$$
\begin{gathered}
\mathrm{J}=P_{e}+\lambda\left(P_{S}+P_{R}-P_{T}\right) \\
\mathrm{J}=N_{0}^{2}\left(\frac{1}{P_{S}{ }^{2} \sigma_{S D}^{2} \sigma^{2}{ }_{S R}}+\frac{1}{P_{S} P_{R} \sigma^{2}{ }_{S D} \sigma^{2}{ }_{R D}}\right) \\
+\lambda\left(P_{S}+P_{R}-P_{T}\right)
\end{gathered}
$$

Now taking the partial derivatives of the Lagrange function $\mathrm{J}$ $(\mathrm{P}, \lambda)$, with respect to $\mathrm{P}_{\mathrm{s}}, \mathrm{P}_{\mathrm{R}}$ and $\lambda$. Afterwards these derivatives will be equated to zero, like defined below

$$
\begin{gathered}
\frac{\partial \mathrm{J}}{\partial P_{S}}=N_{0}^{2}\left[\frac{-2 P_{S} \cdot \sigma^{2} S D \cdot \sigma^{2} S R}{\left(P_{S}^{2} \cdot \sigma^{2} S D \cdot \sigma^{2} S R\right)^{2}}-\frac{P_{R} \cdot \sigma^{2} S D \cdot \sigma^{2}{ }_{R D}}{\left(P_{S} \cdot P_{R} \cdot \sigma^{2} S D \cdot \sigma^{2}{ }_{R D}\right)^{2}}\right]+ \\
\lambda=0 \\
\frac{\partial \mathrm{J}}{\partial P_{R}}=N_{0}^{2}\left[-\frac{P_{S} \cdot \sigma^{2} S D \cdot \sigma^{2}{ }_{R D}}{\left(P_{S} \cdot P_{R} \cdot \sigma^{2} S D \cdot \sigma^{2} R D\right)^{2}}\right]+\lambda=0 \\
\frac{\partial \mathrm{J}}{\partial \lambda}=P_{S}+P_{R}-P_{T}=0
\end{gathered}
$$

After some algebraic manipulations the above equations are used to solve the Power values source and relay node.

$$
\begin{aligned}
& P_{S}=\frac{\sigma_{S R}^{2}+\sqrt{A}}{3 \sigma_{S R}^{2}+\sqrt{A}} P_{T} \\
& P_{R}=\frac{2 \sigma_{S R}}{3 \sigma_{S R}^{2}+\sqrt{A}} P_{T}
\end{aligned}
$$

Where, in the above equation $\mathrm{A}=\left(\sigma_{S R}^{2}+8 \sigma_{R D}^{2}\right)$

\section{B. 3-Hop Scenario}

Similarly, the probability of bit error for the 3-hop scenario will be also be calculated using Moment Generating Function (MGF) approach.. As discussed earlier the moment generating function approach will define the error probability for the particular modulation scheme as an exponential function of $\gamma$, defined below.

$$
\begin{gathered}
P_{e}=\frac{1}{\pi} \int_{0}^{\left(\frac{N-1}{N}\right) \pi} \prod_{n=0}^{1} N_{\gamma_{n}}\left(\frac{g_{P S K}}{\sin ^{2}}\right) d \theta \\
N_{\gamma_{n}}\left(\frac{1}{\sin ^{2}}\right)=\left(1+\frac{g_{P S K}}{\sin ^{2}} \gamma_{n}\right)^{-1} \\
P_{e}=\frac{3 N_{0}^{2}}{8}\left[\frac{P_{e}=\frac{3}{8}\left(\gamma_{0} \cdot \gamma_{1}\right)^{-1}}{P_{S} \cdot \sigma^{2}{ }_{S D} \cdot \sigma_{S R 1}^{2}}+\frac{1}{P_{S} \cdot P_{1} \cdot \sigma_{S D}^{2} \cdot \sigma_{R 1 R 2}^{2}}\right. \\
\left.+\frac{1}{P_{S} \cdot P_{2} \cdot \sigma_{S D}^{2} \cdot \sigma_{R 2 D}^{2}}\right]
\end{gathered}
$$

As the outage probability has been formulated for this 2 relay configuration, now this configuration will be expressed as an optimization problem with transmission power as a constraint entity

$$
\text { Minimize } P_{e}=\frac{1}{\pi} \int_{0}^{\frac{\pi}{2}} \prod_{n=0}^{2} N_{\gamma_{n}}\left(\frac{1}{\sin ^{2}}\right) d \theta
$$$$
\text { Subject to } P_{S}+\sum_{1}^{N} P_{R} \bullet P_{T}
$$

Now as we have established the constrained optimization problem, a closed form expression of this optimization problem will be found using Lagrange Method. The Lagrange cost function of this problem is defined as:

$$
\begin{gathered}
\mathrm{J}=P_{e}+\lambda\left(P_{S}+\sum_{1}^{2} P_{R}-P_{T}\right) \\
\mathrm{J}=\frac{3 N_{0}^{2}}{8}\left(\frac{1}{P_{S}{ }^{2} \sigma_{S D}^{2} \sigma^{2}{ }_{S R 1}}+\frac{1}{P_{S} P_{1} \sigma_{S D}^{2} \sigma^{2} R 1 R 2}\right. \\
\left.+\frac{1}{P_{S} P_{2} \sigma^{2} \sigma_{S D} \sigma_{R 2 D}^{2}}\right)+\lambda\left(P_{S}+P_{1}+P_{2}-P_{T}\right)
\end{gathered}
$$


Now taking the partial derivatives of the Lagrange function $\mathrm{J}$ $(\mathrm{P}, \lambda)$, with respect to $\mathrm{P}_{\mathrm{s}}, \mathrm{P}_{1}, \mathrm{P}_{2}$ and $\lambda$. Afterwards these derivatives will be equated to zero, like defined below

$$
\begin{aligned}
& \frac{\partial \mathrm{J}}{\partial P_{S}}=\frac{3 N_{0}^{2}}{8}\left[\frac{-2 P_{S} \cdot \sigma^{2} S D \cdot \sigma^{2} S R 1}{\left(P_{S}^{2} \cdot \sigma^{2} S D \cdot \sigma^{2} S R 1\right)^{2}}-\frac{P_{1} \cdot \sigma^{2} S D \cdot \sigma_{R 1 R 2}^{2}}{\left(P_{S} \cdot P_{1} \cdot \sigma^{2} S D \cdot \sigma_{R 1 R 2}^{2}\right)^{2}}-\right. \\
& \left.\frac{P_{2} \cdot \sigma^{2} S D \cdot \sigma_{R 2 D}^{2}}{\left(P_{S} \cdot P_{2} \cdot \sigma^{2}{ }_{S D} \cdot \sigma^{2}{ }_{R 2 D}\right)^{2}}\right]+\lambda=0 \\
& \frac{\partial \mathrm{J}}{\partial P_{1}}=\frac{3 N_{0}^{2}}{8}\left[\frac{-P_{S} \cdot \sigma_{S D}^{2} \cdot \sigma_{R 1 R 2}^{2}}{\left(P_{S} P_{1} \cdot \sigma_{S D}^{2} \cdot \sigma_{R 1 R 2}^{2}\right)^{2}}\right]+\lambda=0 \\
& \frac{\partial \mathrm{J}}{\partial P_{2}}=\frac{3 N_{0}^{2}}{8}\left[\frac{-P_{S} \cdot \sigma_{S D}^{2} \cdot \sigma_{R 2 D}^{2}}{\left(P_{S} P_{2} \cdot \sigma_{S D}^{2} \cdot \sigma_{R 2 D}^{2}\right)^{2}}\right]+\lambda=0 \\
& \frac{\partial \mathrm{J}}{\partial \lambda}=P_{S}+P_{1}+P_{2}-P_{T}=0
\end{aligned}
$$

.After some algebraic manipulations the above equations are used to solve the value of $\mathrm{P}_{\mathrm{S}}, \mathrm{P}_{1}$ and $\mathrm{P}_{2}$.

$$
\begin{gathered}
P_{S}=\left\{\begin{array}{c}
\frac{A-4 B+\sqrt{A^{2}+8 A B}}{4(A-B)} P_{T} \\
\frac{2}{3} P_{T}
\end{array}\right. \\
P_{1}=\frac{\sigma_{2 D}}{\sigma_{2 D}+\sigma_{R 1 R 2}}\left(P_{T}-P_{S}\right) \\
P_{2}=P_{T}-P_{1}-P_{S}
\end{gathered}
$$

Where, in the above equation $\mathrm{A}=\left(\sigma_{R 2 D}+\sigma_{R 1 R 2}\right)^{2} \cdot \sigma_{R 1 R 2}^{2} \cdot \sigma^{2}{ }_{S R 1}$ and $\mathrm{B}=\sigma_{R 2 D}^{2} \cdot \sigma^{3}{ }_{R 1 R 2}$

\section{SIMULATION RESULTS}

Fig. 3, compares the performance of OPA with EPA in terms of ABER for a cooperative network having 2 relays. The results show that OPA clearly enhances the system performance.

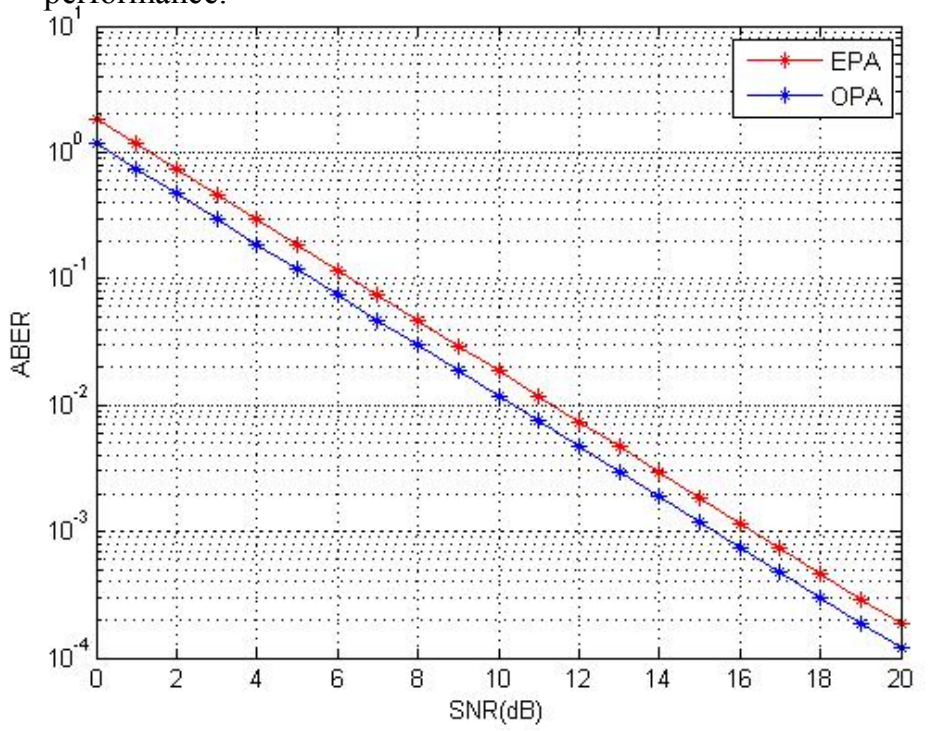

Figure 3. Equal power allocation and Optimal power allocation

\section{A. Results (2-Hop Scenario)}

Fig. 4, compares the performance of case 1: $\left(\sigma_{S D}^{2}=\right.$ 1; $\left.\sigma_{S R}^{2}=1 ; \sigma_{R D}^{2}=1\right)$ with case $2:\left(\sigma_{S D}^{2}=1 ; \sigma_{S R}^{2}=\right.$ $\left.1 ; \sigma_{R D}^{2}=10\right)$ in terms of ABER for a cooperative network having a single relay. Here, channel link quality $\left(\sigma^{2}\right)$ is denoted by 10 if the two communicating nodes are closer to each other while if the two nodes are far from each other channel link quality is 1 . The graph show that case 2 is performing marginally better than case 1, when in lower SNR ranges $0-5(\mathrm{dBs})$ and the performance gradually increases with increased SNR. The reason is that cooperative relaying performs better when the link between last relay and destination node is strong [9]. Moreover, as cooperative relaying employs a receiver diversity technique like MRC in our case, the system performance is increased if the incoming signal being combined comes from a stronger channel.

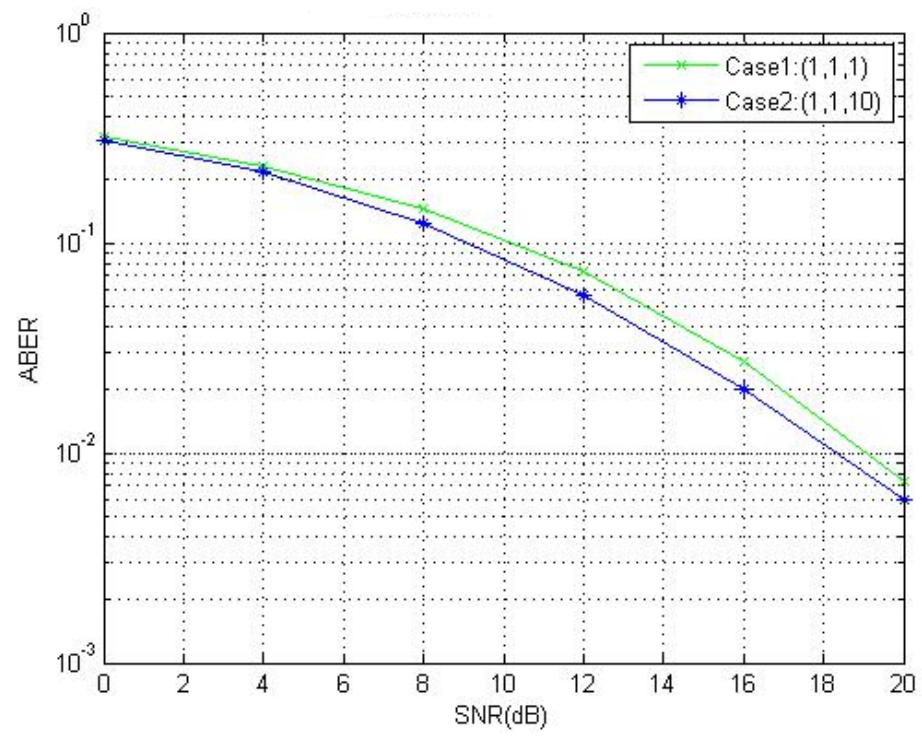

Figure 4. Power allocation with AF involving 1-Relay

\section{B. Results (3-Hop Scenario)}

Fig. 5, compares the performance of OPA with 6 different scenarios in terms of ABER for a cooperative network having 2 relays. In the graph, case $3:\left(\sigma_{S D}^{2}=1 ; \sigma_{S R 1}^{2}=\right.$ 10; $\left.\sigma_{R 1 R 2}^{2}=10 ; \sigma_{R 2 D}^{2}=10\right)$ will be the ideal scenario where both of the relying nodes lie in closer proximity to the destination node and has clearly the best performance curve and case 1: $\left(\sigma_{S D}^{2}=1 ; \sigma_{S R 1}^{2}=1 ; \sigma_{R 1 R 2}^{2}=1 ; \sigma_{R 2 D}^{2}=1\right)$ is the worst performance curve having no stronger paths. As we have established in the earlier scenario (2-hop) that the system perfromance depends a lot on the channel link quality $\left(\sigma^{2}\right)$ between source and the first relay and the link quality between the destination node and the last relay. This is being depicted from the performance curves of case $2:\left(\sigma_{S D}^{2}=\right.$ $\left.1 ; \sigma_{S R 1}^{2}=1 ; \sigma_{R 1 R 2}^{2}=1 ; \sigma_{R 2 D}^{2}=10\right)$ and case $5:\left(\sigma_{S D}^{2}=\right.$ $\left.1 ; \sigma_{S R 1}^{2}=10 ; \sigma_{R 1 R 2}^{2}=1 ; \sigma_{R 2 D}^{2}=1\right)$. Moreover, both case 4: $\quad\left(\sigma_{S D}^{2}=1 ; \sigma_{S R 1}^{2}=1 ; \sigma_{R 1 R 2}^{2}=10 ; \sigma_{R 2 D}^{2}=10\right)$ case 6: $\quad\left(\sigma_{S D}^{2}=1 ; \sigma_{S R 1}^{2}=10 ; \sigma_{R 1 R 2}^{2}=1 ; \sigma_{R 2 D}^{2}=10\right)$ have better performance than case 2 and case 5 because both of them have more than one strong path. 


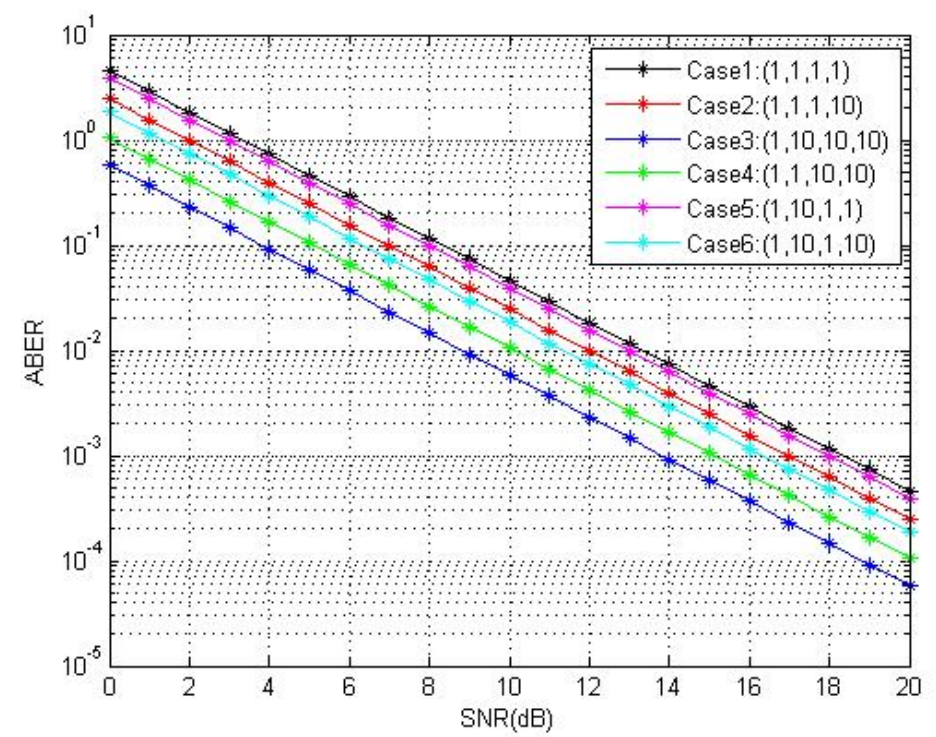

Figure 5. Power allocation with AF involving 2-Relays

The power allocation algorithm optimally allocates transmission power among source node and the involved relay nodes. The power is calculated based on the channel link qualities between the two communicating nodes. In Table I, the values of transmission powers of the nodes for 2-hop configuration are presented. Here, if the relay node is closer to the source node almost equal power is allocated to both of them. When the relay node is closer to the destination node, source node is allocated more power [9].

TABLE I. OPTIMAL POWER VALUES (2-Hop CONFIGURATION)

\begin{tabular}{|l|l|l|}
\hline $\boldsymbol{\sigma}^{2}{ }_{S D} ; \boldsymbol{\sigma}^{2}{ }_{S R} ; \boldsymbol{\sigma}^{2}{ }_{R D}$ & $\begin{array}{l}\text { Source } \\
\text { Node }\left(\boldsymbol{P}_{S}\right)\end{array}$ & $\begin{array}{l}\text { Relay } \\
\text { Node }\left(\boldsymbol{P}_{\boldsymbol{I}}\right)\end{array}$ \\
\hline$(1,1,1)$ & 0.6667 & 0.3333 \\
\hline$(1,1,10)$ & 0.8187 & 0.1813 \\
\hline$(1,10,1)$ & 0.5472 & 0.4528 \\
\hline
\end{tabular}

In Table II, the transmission powers values of the nodes for a 3-hop configuration are presented. The power values are calculated from the formulae derived earlier in Section-III, keeping in mind the constraint of transmission power.

TABLE II. OPtimal POWER VALUES (3-Hop CONFIGURATION)

\begin{tabular}{|l|l|l|l|}
\hline$\sigma_{S D}^{2} \sigma_{S R 1}^{2} \sigma_{R 1 R 2}^{2} \sigma_{R 2 D}^{2}$ & $\begin{array}{c}\text { Source } \\
\text { Node (P) }\end{array}$ & $\begin{array}{c}\text { Relay 1 } \\
\text { Node (P) }\end{array}$ & $\begin{array}{c}\text { Relay 2 } \\
\text { Node }\left(P_{\nu}\right)\end{array}$ \\
\hline$(1,1,1,10)$ & 0.6236 & 0.2778 & 0.0986 \\
\hline$(1,10,10,10)$ & 0.5774 & 0.2113 & 0.2113 \\
\hline$(1,1,10,10)$ & 0.7186 & 0.1407 & 0.1407 \\
\hline$(1,10,1,1)$ & 0.5144 & 0.2428 & 0.2428 \\
\hline$(1,10,1,10)$ & 0.5288 & 0.3478 & 0.1234 \\
\hline
\end{tabular}

Here, it can be seen that if the channel link quality between any two communicating nodes is strong, comparatively less power will be assigned to the transmitting node. On the other hand if the channel link quality is weak then more power is assigned to the corresponding transmitting node

\section{CONCLUSIONS}

Simulation results show that optimizing the power allocation is essential for cooperative networks having unbalanced channel link qualities. For a multi-hop cooperative network, the most significant channel link qualities are of the paths between source and the first relay node and that of between the last relay and the destination node. The effect of channel link qualities of the other paths on performance of the system is considerably small. Moreover, if more than one path is strong during a multi-hop cooperative transmission, the receiver diversity technique will help the system to achieve better performance. Moreover, as a future direction if opportunistic relaying is employed, the system performance can improve further.

\section{REFERENCES}

[1] J. N. Laneman, G. W. Wornell. Distributed space-time coded protocols for exploiting cooperative diversity in wireless networks [J]. IEEE Trans. on Information Theory, 2003, 49(10): $2415-2425$.

[2] J. N. Laneman, David N. C. Tse, G. W. Wornell. Cooperative diversityin wireless network: efficient protocols and outage behavior [J]. IEEE Trans. on Information Theory, 2004, 50(12): 3062-3080.

[3] Weifeng Su, Ahmed K.Sadek and K.J.Ray Liu. Cooperative communication protocols in wireless networks: performance analysis and optimum power allocation [J]. Wireless Personal Commun., 2008, 44(2): 181-217.

[4] Yan Wang, Fei Lin. SEP performance analysis and power allocation for amplify-and-forward relay networks [C]. // The 3rd IEEE International Conference on Wireless Communications, Networking and Mobile Computing, September 21-23, 2007, Shanghai China (Wicom 2007):12741277.

[5] Mulugeta K. Fikadu, Mohammed Elmusrati, and Reino Virrankoski, Power Allocation in Multi-node Cooperative Network in Rician Fading Channels. IEEE 8th International Conference on Wireless and Mobile Computing, Networking and Communications (WiMob) 2012: 496-501

[6] Sarmad Sohaib and Daniel K. C. So, Power Allocation for Multi-Relay Amplify-and-Forward Cooperative Networks. IEEE Conference on Communications (ICC), 2011: 1-5

[7] Nan Zhang, Jian Hua Ge and Feng Kui Gong, SEP analysis and optimal power allocation of multinode amplify-and-forward cooperation systems. $2008,1-5$

[8] Jia Liu, Ness B. Shroff, and Hanif D. Sherali. Optimal Power Allocation in Multi-Relay MIMO Cooperative Networks: Theory and Algorithms. IEEE Journal on Selected Areas in Communications. 2012, 30(2): 331-340

[9] Ahmed K.Sadek, Weifeng $\mathrm{Su}$ and K.J.Ray Liu. .Multinode Cooperative Communications in Wireless Networks. Signal Processing, IEEE Transactions on. 2007, 55(1): 341-355. 
Muhammad H. D. Khan (S'13) was born in Rawalpindi, Pakistan, in 1986. He received the B.E. degree in electrical engineering from the COMSATS Institute of Information Technology, Islamabad, Pakistan, in 2010, and the M.Sc. degree in technology from University of Vaasa, Vaasa, Finland, in 2013.In 2010, he joined the Department of Avionics Engineering, Air University, Pakistan, as a Research Assistant, and in 2011 became a Research Associate. His current research interests include wireless communication systems, sensor networks and resource allocation management.

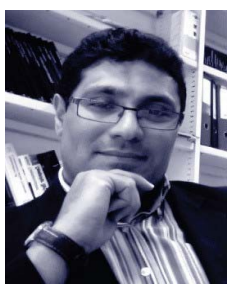

Mohammed Elmusrati (S'00-M'04-SM'12) received the B.Sc. (with honors) and M.Sc. (with high honors) degrees in telecommunication engineering from the Electrical and Electronic Engineering Department, Benghazi University, Libya, in 1991 and 1995, respectively, and the Licentiate of Science in technology (with distinction) and the Doctor of Science in Technology degrees in control engineering from Aalto University - Finland, in 2002 and 2004, respectively. Currently, Elmusrati is full professor and head of communications and systems engineering group at University of
Vaasa - Finland. His main research interests include Radio resource management in wireless communication, wireless networked control, game theory, and smart grids.

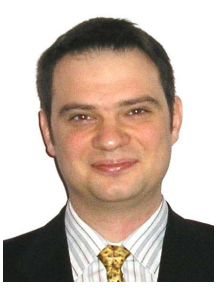

Reino Virrankoski received his M.Sc degree from the University of Helsinki on 2000 and he is a Ph.D.student in Control Engineering at Helsinki University of Technology. On 2000-2007 he was working as a Graduate Researcher at Helsinki University of Technology. During that time he also worked as a Visiting Assistant Researcher at Yale University on 2004-2005. Currently he holds a faculty position as a Lecturer of Telecommunications at the University of Vaasa. Mr. Virrankoski's main research interests are communication and control in telecommunication systems and in wireless sensor networks, wireless automation, localization and controlled mobility and wireless networks in defense and security. 